Cinémas

Revue d'études cinématographiques

Journal of Film Studies

\title{
Barry Lindon démembré : la perte de l'histoire dans le film de Stanley Kubrick
}

\section{Lara Fitzgerald et Christopher James Keep}

Volume 4, numéro 1, automne 1993

Écrit/Écran

URI : https://id.erudit.org/iderudit/1000108ar

DOI : https://doi.org/10.7202/1000108ar

Aller au sommaire du numéro

Éditeur(s)

Cinémas

ISSN

1181-6945 (imprimé)

1705-6500 (numérique)

Découvrir la revue

Citer cet article

Fitzgerald, L. \& Keep, C. J. (1993). Barry Lindon démembré : la perte de l'histoire dans le film de Stanley Kubrick. Cinémas, 4(1), 23-33.

https://doi.org/10.7202/1000108ar
Résumé de l'article

Le film Barry Lyndon (1975) de Stanley Kubrick est peut-être l'oeuvre le moins appréciée du directeur américain. Reconnu pour sa valeur artistique, les critiques, néanmoins, condamnèrent Barry Lyndon pour l'opulence exorbitante du décor et pour " sa technique trop perfectionnée ». La présente étude propose de réexaminer l'excès de la méthode de Kubrick. Dans un premier temps, les auteurs établissent de quelle façon Kubrick s'abstient des conventions du cinéma réaliste afin de révéler l'altérité radicale du passé, et, par ailleurs, comment son obsession du détail historique finit par nous éloigner et non pas nous rapprocher du XVIII ${ }^{\mathrm{e}}$ siècle. Étant donné cette impossibilité de re-présenter l'histoire dans la plénitude du présent, les auteurs examinent, dans un deuxième temps, comment Kubrick montre la violence du signe cinématographique. Dans Barry Lyndon, Kubrick opère une " inversion catastrophique " où la proximité de l'événement à l'écran si familière du cinéma réaliste est ressentie comme une intrusion étrange, comme une violente pénétration de l'espace. 


\title{
Barry Lyndon démembré : la perte de l'histoire dans le film de Stanley Kubrick ${ }^{1}$
}

\section{Lara Fitzgerald et Christopher James Keep}

\begin{abstract}
RÉSUMÉ
Le film Barry Lyndon (1975) de Stanley Kubrick est peut-être l'œuvre le moins appréciée du directeur américain. Reconnu pour sa valeur artistique, les critiques, néanmoins, condamnèrent Barry Lyndon pour l'opulence exorbitante du décor et pour «sa technique trop perfectionnée». La présente étude propose de réexaminer l'excès de la méthode de Kubrick. Dans un premier temps, les auteurs établissent de quelle façon Kubrick s'abstient des conventions du cinéma réaliste afin de révéler l'altérité radicale du passé, et, par ailleurs, comment son obsession du détail historique finit par nous éloigner et non pas nous rapprocher du XVIIIe siècle. Étant donné cette impossibilité de re-présenter l'histoire dans la plénitude du présent, les auteurs examinent, dans un deuxième temps, comment Kubrick montre la violence du signe cinématographique. Dans Barry Lyndon, Kubrick opère une «inversion catastrophique» où la proximité de l'événement à l'écran si familière du cinéma réaliste est ressentie comme une intrusion étrange, comme une violente pénétration de l'espace.
\end{abstract}

\footnotetext{
ABSTRACT

The film Barry Lyndon (1975) by Stanley Kubrick is perhaps the least appreciated work by this American director. Though recognizing its artistic quality, critics nevertheless condemned Barry Lyndon for the exorbitant opulence of its decor and its "overly perfect technique." This study is a reexamination of excess in Kubrick's method. First the authors establish how Kubrick evades the conventions of realist cinema in order to reveal the
} 
radical otherness of the past and how his obsession with historical detail ends up distancing us from the eighteenth century rather than bringing us closer. Given the impossibility of re-presenting history with the fullness of the present, the authors secondly investigate how Kubrick displays the violence of the cinematographic sign. In Barry Lyndon, Kubrick executes a "catastrophic inversion" where the proximity of the events on the screen, so familiar in realist cinema, is experienced as a strange intrusion, a violent penetration of space.

On a parlé du film Barry Lyndon de Stanley Kubrick comme du «film expérimental le plus cher jamais réalisé» (Stephenson, p. 259, nous traduisons). Un critique l'a même qualifié «d'interminable présentation de diapositives pour les étudiants en histoire de l'art» (Feldman, p. 12, nous traduisons). Pour ses critiques, Barry Lyndon en fait trop : trop long, trop lent, trop formel, trop technique; trop de décors, trop d'artifice ou alors... trop réel. Par son opulence exorbitante, Barry Lyndon semble remplir au point de faire déborder la vaste capacité du réservoir de l'épopée historique.

Cette étude se propose une lecture de l'excès de la méthode Kubrick comme symptôme. Le coût de production peut être considéré non plus en termes de millions de dollars dépensés sur le film (onze), mais plutôt à travers la thématisation de la notion même de production. La tentative pour récupérer le perdu, pour re-présenter l'histoire dans la plénitude du présent possède, en fait, son propre coût. Cette tentative de récupération s'autoépuise, frustrant ainsi le désir spectatoriel de connaître «le passé». Kubrick enfonce un coin entre passé et présent, entre le signifiant filmique et son référent historique afin d'effectuer une réflexion critique sur les concepts du temps et de l'espace dans le cinéma; afin, aussi, de scruter la violence à partir de laquelle le cinéma crée l'illusion de proximité. L'obsession du détail historique ainsi que l'attention méticuleuse au costume et au décor d'époque portent en elles la marque de la perte. Cette perte est cependant une perte productive; c'est elle qui ouvre, à nouveau, l'espace de l'histoire.

Le film Barry Lyndon est une adaptation très approximative du roman de William Makepiece Thackerey The Luck of Barry Lyndon, publié en 1844. Le roman retravaille une histoire réelle : celle de l'essor et de la chute d'un arriviste irlandais. Dans le roman et dans le film, on suit les aventures de Redmond 
Barry, personnage qui accède au monde des aristocrates grâce à son charme et à son caractère déceptif. Afin de consolider sa position dans la haute société, Barry épouse la belle veuve Lady Lyndon, une noble extrêmement riche, dont il dissipe bientôt la fortune. Pourtant, Lord Bullingdon, fils du premier mariage de Lady Lyndon, finit par l'exiler du château familial. Le film est si différent du roman (par exemple, le point culminant, le duel entre Bullingdon et Barry, a été inventé expressément pour le film) qu'un critique a remarqué que «Kubrick s'est tellement écarté du roman, qu'on se demande pourquoi il a d'abord décidé de l'adapter» (Feldman, p. 12, nous traduisons).

Cette distance entre roman et film annonce déjà la distance que la caméra maintient par rapport à l'action. La caméra de Kubrick est, pour la plupart, immobile; lorsqu'il y a mouvement, les panoramiques et les travellings sont très lents. La fréquence des gros plans et des zooms arrière réduit les personnages à des figures minuscules. Par ailleurs, la caméra s'attarde souvent sur la scène, prolongeant ainsi la lenteur et la majesté du film; et le cadre pittoresque des plans moyens et des plans rapprochés est si soigneusement composé qu'il reproduit la manière des portraits de l'époque. Chaque nouvelle scène est introduite par un plan formel qui présente le lieu de l'action à venir. Ces plans-ci mettent en valeur non seulement la nature grandiose de l'architecture, mais encore ils insistent sur le caractère inabordable de ces lieux qui appartiennent au passé (Stephenson, p. 258).

Selon Frederic Jameson, la distance que Kubrick instaure entre passé et présent dans Barry Lyndon évacue le sens de l'histoire; c'est un processus qui, pour Jameson, coïncide avec la montée du capitalisme. Le monde hermétiquement scellé de Barry Lyndon entrave donc la possibilité d'envisager la fin du XVIII e siècle comme le précurseur du XXe siècle. Ce dispositif prive le film de toute motivation :

Le paradoxe, le mystère historique de la dévitalisation de la forme sera ressenti par les gens pour qui le film Barry Lyndon, avec ses images éclatantes et son interprétation extraordinaire, est en quelque sorte, profondément gratuit; un objet flottant dans le vide qui aurait pu tout aussi bien ne pas exister; sa technique est trop perfectionnée pour n'être qu'un simple exercice formel, et pourtant, sa non-justification est profondément inquiétante (Jameson, p. 243, nous traduisons).

Ce commentaire de Jameson fait écho à l'argument de Georges Lukacs quant au déclin du roman historique. Dans son livre Le Roman historique, le philosophe prétend que le genre s'est épuisé 
avec Salammbô de Flaubert. C'est de la tentative de recréer Carthage selon un détail historique exact que découlerait la disjonction radicale entre la vie intérieure des personnages et le monde dans lequel ils sont censés vivre. Par exemple, selon Lukacs, le dilemme psychologique de Salammbô provient moins des conditions matérielles de Carthage au IVe siècle, que de la condition de la femme bourgeoise française au XIX ${ }^{\mathrm{e}}$ siècle : Salâmmbo est en fait une sorte de Madame Bovary emprisonnée dans une histoire étrangère. Ainsi, le roman de Flaubert devient «un monde de costumes et de décors, historiquement exacts, rien de plus qu'un cadre pittoresque, au sein duquel se déroule un processus purement moderne» (Lukacs, p. 211).

Pour Jameson, comme pour Lukacs, l'impression qu'il existe une division entre la vie intérieure et la vie extérieure des personnages est un symptôme de la perte de la continuité de l'histoire, c'est-à-dire de la perte du sens de l'historicité. Jameson prétend dès lors que «le sens de ce moment déterminant» - la fin du XVIIIe siècle - «est un moment de nécessité fondamentale, un précurseur du présent disparu dans le pluralisme du musée imaginaire» (Jameson, p. 243, nous traduisons). Or, c'est précisément cette perte que Kubrick cherche à explorer : cette distance délibérée qui caractérise le film, distance qui écarte le spectateur de la diégèse, qui sépare le XVIII ${ }^{e}$ siècle du $\mathrm{XX}^{\mathrm{e}}$, n'est pas comme le voudrait Jameson, un effet secondaire du film, mais elle constitue «l'objet même» d'étude.

La caméra détachée de Kubrick met au premier plan les moyens par lesquels le signe cinématographique construit le temps et l'espace. Le caractère visuel de l'image se prête particulièrement à la description empirique par laquelle le roman historique essaye d'actualiser. Un passage de plusieurs pages, où Thackeray décrit, par exemple, le domaine de la famille Lyndon, est présenté à l'écran en un seul plan. Certes, nous avons ici affaire à la nature polysémique de l'image, à «l'épaisseur de signes de l'expression cinématographique» barthésienne (Barthes, p. 106). Nous avons aussi, par ailleurs, une deuxième illusion, celle de la plénitude de la présence : «L'image filmique serait au présent parce qu'elle nous donnerait l'impression de suivre cette action "en direct"»(Gaudreault et Jost, p. 101). L'image actualise donc un passé, des événements révolus. Comme nous le rappelle Barthes, l'image cinématographique est «un leurre parfait» (p. 104) qui «peut produire le retentissement de la vérité» (p. 106) - ce qui crée l'illusion d'une présence pleine. Le signe cinématographique tend donc à produire l'illusion du 
passé se déroulant devant nos yeux pour la première fois. Or, Kubrick fait contrepoids à cet effet du cinéma en le dévoilant justement comme effet. Le cinéaste présente ainsi son film comme une action révolue, comme une action qui appartient entièrement au passé.

Le roman de Thackerey est raconté à la première personne par Redmond Barry. L'arriviste irlandais, qui est d'une famille douteuse, raconte son histoire afin d'affirmer sa réputation en tant qu'héritier «de la famille la plus noble de l'île, et peut-être la plus noble du monde entier» (p. 4, nous traduisons). Le roman nous offre un accès immédiat aux pensées et aux sentiments du narrateur; nous sommes placés dans la conscience de Barry et lors du déroulement de sa narration, son caractère "véritable» se révèle à nous, lecteurs. Le monologue de Barry prend la forme d'un dévoilement involontaire; dans la tentative de présenter une apologie convaincante, Barry se trahit : il se révèle vantard et menteur.

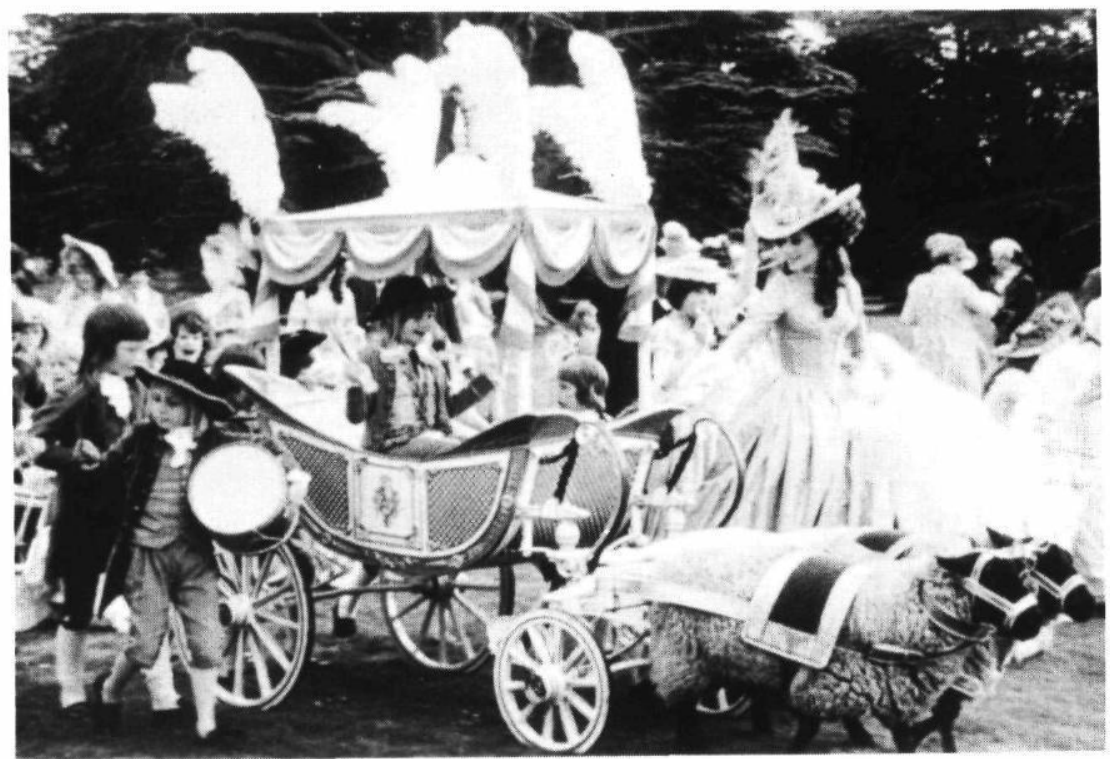

Barry Lyndon de Stanley Kubrick

Coll. Cinémathèque québécoise 
Le film Barry Lyndon, par contre, nous offre une expérience entièrement différente. Au lieu de nous trouver dans l'esprit du protagoniste, nous restons résolument à l'extérieur. Le narrateur de Kubrick parle en voix over, à la troisième personne. Distanciée des événements à l'écran, la voix over raconte l'histoire d'un ton ironique et détaché. Tout au long du film, la voix intervient pour annoncer des événements à suivre, annulant toute possibilité de suspense. On apprend, par exemple, la mort du petit Bryan bien avant qu'elle ne nous soit montrée. Par ailleurs, lors des scènes d'émotion ou de splendeur visuelle, la voix intervient afin de réduire toute intensité de l'instant à l'écran, de nous distancier de l'histoire. Le narrateur aplatit ainsi toute tension dramatique, et donne au récit le sens d'un fait accompli. L'illusion que les événements sont en cours, qu'ils se déroulent devant nos yeux nous est constamment dérobée. Le spectateur est conscient du fait qu'il s'agit d'un récit déjà accompli, enfermé dans les archives de l'histoire.

Le cadre pittoresque de la mise en scène de Kubrick, qui distancie le spectateur de la diégèse, souligne ainsi l'extériorité du signe cinématographique. L'image qui impressionne la pellicule est en mesure de rendre présent ce qui ne l'est pas, de re-présenter le passé dans un détail "vivant», mais ce ne peut être qu'une illusion, qu'un effet de nature polysémique. Par le biais d'une batterie de techniques narratives et filmiques, le cinéma réaliste traditionnel crée un effet de suture entre le spectateur et le point de vue du protagoniste. C'est à cette fin qu'y sont construits les point of view shots, les raccords sur le regard et sur le mouvement, la narration à la première personne en voix off, bref, un engagement général à l'idéologie de l'individu : et c'est de ces conventions filmiques que Barry Lyndon s'abstient. En luttant délibérément contre l'apparente plénitude du signe cinématographique, le film frustre les attentes de l'épopée historique qu'entretient son spectateur. Barry Lyndon refuse tout sentiment de participation, afin de mettre en valeur l'éloignement, voire l'altérité extrême du passé.

Tenu à l'écart des événements et des personnages à l'écran, le désir de connaître les personnages du film est donc constamment contrecarré. La plupart des personnages nous semblent artificiels, des êtres en celluloïd qui ressemblent à des mannequins. Kubrick s'abstient de créer des profils psychologiques pour ses personnages, et le spectateur éprouve ainsi énormément de difficulté à connaître leurs pensées et leurs motivations. L'intensité émotionnelle des scènes est toujours dissoute. La scène d'amour entre Barry et Lady Lyndon nous 
fournit un exemple : c'est une scène longue, lentement filmée où on voit les amoureux échanger des regards lors d'un jeu de cartes. Cependant, les figures oniriques de Barry et de Lady Lyndon sont si opaques et leurs manières sont tellement rigides que les expressions de leurs visages ne trahissent aucune émotion. Le manque de plans rapprochés maintient la distance émotionnelle filmique. Le point culminant - qui, dans une scène d'amour, est d'habitude très affectif et passionné - est ici un petit baiser entre les amoureux. Par ailleurs, toute interprétation spectatorielle de la scène qui comprend l'opacité comme une sorte d'intensité émotionnelle est rapidement dissipée par la scène suivante où la voix over, sarcastique, annonce que Lady Lyndon est tombée amoureuse de Barry en moins de six heures.

Le passé, nous suggère le film, n'est donc récupérable que lors de sa disparition perpétuée dans l'abondance polysémique de l'image. La distance avec laquelle Kubrick examine son matériel historique s'avère, d'une part, une tentative calculée pour adapter à l'écran le sens des convenances et l'équilibre qui caractérise les portraits néo-classiques couvrant les murs du château Lyndon. Mais, comme le remarque Jean-Pierre Oudart, l'hyperréalisme de Kubrick opère d'autre part «une inversion catastrophique» ( $p$. 62). Par une ruse particulière de la perspective - ruse qui constitue en fait le signe cinématographique -, on s'aperçoit que plus on s'approche de l'histoire, plus l'histoire nous échappe. Par la tentative même de faire vivre le détail et la sensibilité esthétique de «l'âge de la Raison», le cinéaste surcharge le cadre et, paradoxalement, il renforce le sentiment d'un éloignement irrémédiable. Diviser la chose d'elle-même, disperser la présence unique, celle que la représentation s'efforce de reprendre à l'abîme de l'histoire, tel est l'effet de ce que Jacques Derrida appelle «la violence originaire du langage» :

(...) la violence originaire du langage (...) consiste à inscrire dans une différence, à classer, à suspendre le vocatif absolu. Penser l'unique dans le système, l'y inscrire, tel est le geste de l'archiécriture : (...) de la proximité absolue, de la présence à soi, perte en vérité de ce qui n'a jamais eu lieu, d'une présence à soi qui n'a jamais été donnée mais rêvée et toujours dédoublée, répétée, incapable de s'apparaître autrement que dans sa propre disparition (Derrida, 1967 : pp. 164-165).

L'abondance polysémique de l'image, que nous avons caractérisée comme inhérente à celle-ci, est, pour Derrida, la condition de toute écriture. Pourtant, cette abondance a été supprimée par l'époque du logocentrisme : «le mirage de la chose même, de la présence immédiate de la perception» est à la 
fois produit et différé par «l'enchaînement infini» de signifiants qui indiquent non pas des signifiants spécifiques, mais d'autres signifiants. L'écriture, selon Derrida, n'est pas l'inscription graphique qui exprime la parole, mais une forme d'espacement, de mouvement et de jeu. Cette écriture, pour lui, devient «dissémination», processus par lequel il existe toujours un reste qui dépasse le sens, une certaine perte, «une semence dépensée au-dehors en pure perte» - celle qui ouvre le jeu de la différence (Derrida, 1972 : p. 172).

Le caractère exorbitant de Barry Lyndon, son excès technique et décoratif mènent en effet à une perte : perte de proximité à la chose en elle-même, perte d'intériorité, perte d'engagement. Ce film inscrit ce sens de la perte non seulement dans la qualité picturale de la mise en scène, mais également au niveau de la narration. La tentative de Barry Lyndon pour gagner le respect de la société, pour suppléer les défauts de son milieu familial est marquée par une série de défaites, de coûts, de réductions. Nora enlevée par le captaine Quin, Barry perd son premier amour et des bandits de grand chemin lui volent les maigres économies de sa mère. Soldat caché à l'étranger, Barry est ainsi coupé de son pays natal et, lors de son séjour forcé dans l'armée prusse, il y est humilié par les autres soldats; ses valeurs morales sont minées. Lors de sa liaison avec le chevalier de Balibari, Barry Lyndon voit sa vie réduite à celle d'un criminel charmant.

Même le plus grand succès de Barry, son mariage avec Lady Lyndon, se termine par une série de rabaissements. Le mariage est vide et peu satisfaisant et le seul lien entre Barry et sa femme, leur fils Bryan, disparaît lors d'un accident de cheval - le cadeau empoisonné du père. Sa tentative de s'acheter un titre de noblesse, de se procurer une nouvelle identité, un nom différent échoue aussi. Il se paie des fêtes coûteuses pour plaire aux aristocrates, il s'achète des tableaux et des vêtements luxueux afin de se faire passer pour «un homme de qualité», et c'est ainsi que Barry épuise la fortune considérable de sa femme. À la fin du film, son némésis Lord Bullingdon triomphe en refusant de l'appeler par son nom d'adoption de Barry Lyndon; il l'appelle toujours Redmond Barry. Chaque tentative de Barry d'ajouter à sa vie, chaque effort pour remplir l'espace de sa famille absente, finit par échouer. L'acte de production, celle de la tentative de rendre présent ce qui ne l'est pas, entraîne nécessairement un coût, un coût qui éloigne de plus en plus Barry de son but.

Fait révélateur : les moments du film qui nous offrent un sentiment de proximité, de rapprochement de l'action sont les scènes de violence. En fait, c'est à ces moments-là qu'en tant que 
spectateurs nous ressentons, paradoxalement, ce sentiment de perte qui accompagne la plénitude du signe cinématographique. Comme Kubrick l'a remarqué lui-même, Barry Lyndon, malgré toute la finesse artistique et l'étiquette, est un film «extrêmement turbulent» (cité dans Ciment, p. 162).

La violence prend alors deux formes : d'une part, celle des escarmouches orchestrées, contrôlées, des soldats professionnels de la guerre de sept ans; d'autre part, les soudaines explosions de rage de Barry. La première forme conserve le sens des convenances et de la distance qui domine la mise en scène du film. La bataille entre les armées française et anglaise, à laquelle Barry participe, est filmée avec la même distance et à la même allure que la scène d'amour que nous avons commentée plus haut.

La deuxième forme de violence, par contre, ponctue le vernis de l'objectivité de la première. Lorsque Barry attaque Lord Bullingdon parce qu'il a interrompu un concert et a insulté sa famille, la caméra nous met au centre de la mêlée. Les panoramiques lents et les perspectives éloignées ont tout à coup fait place au style de la caméra à main, virant de façon abrupte d'un plan rapproché à un autre plan rapproché. Les airs majestueux qui marquent tellement les convenances, ce sens de l'étiquette qui traverse le film et ces airs qui font, par ailleurs, partie de la diégèse de cette scène sont eux aussi interrompus par la cacophonie soudaine des voix, des cris et des bruits de la dispute.

La violence de Barry Lyndon a un effet étrange, prenant un sens de défamiliarisation que nous ne reconnaissons qu'au moment de sa distorsion. L'attaque de Barry sur Bullingdon donne l'impression d'un événement qui se déroule dans le présent : cette scène a toutes les marques de la proximité, marque de la relation du signe cinématographique au passé du film réaliste. Et pourtant, ce moment-ci est ressenti comme une intrusion, comme une trangression de l'éloignement si soigneusement construit par le film. Cette présence, si familière dans le cinéma réaliste, nous paraît maintenant tout à fait étrangère du fait qu'elle se trouve associée à une violente pénétration de l'espace. En rendant le cinéma étrange, Kubrick nous le dévoile à nouveau. La violence productive du signe cinématographique a été d'abord notée par Walter Benjamin dans son essai «L'EEuvre d'art à l'ère de sa reproductibilité technique». Écrivant en 1936, Benjamin compare le cinéaste et le peintre et note que leur différence est analogue à celle qui distingue le chirurgien du mage : 
L'attitude du mage, qui guérit un malade par l'imposition des mains, diffère de celle du chirurgien qui pratique sur lui une intervention. Le mage conserve exactement la distance naturelle entre lui et le patient. Le chirurgien, au contraire, la diminue considérablement - parce qu'il intervient à l'intérieur du malade - mais il ne l'augmente que peu - grâce à la prudence avec laquelle sa main se meut parmi les organes du patient (Benjamin, p. 112).

Comme le chirurgien, le cinéaste, dit Benjamin, "pénètre profondément dans la trame même de la réalité» (p. 112). Barry Lyndon semble reprendre ces deux positions. La qualité picturale de la mise en scène met en valeur la distance du passé, nous forçant à percevoir son altérité radicale. Mais lors des moments de rage cathartique, le film raccourcit la distance entre la caméra et la diégèse; il pénètre le corps de son sujet, opérant ainsi une perte qui est en même temps la construction du film. La relation entre l'élément pictural des tableaux et l'élément filmique n'est pas, pour Kubrick, une relation d'opposition; elle est une relation de différence. L'un est la condition de l'existence de l'autre, il offre les moyens par lesquels l'espace même de la représentation est ouvert.

Dans la scène du duel entre Barry et Lord Bullingdon, scène qui n'existe pas dans le roman de Thackerey ni dans l'histoire "véritable» d'Andrew Stoney Bowes, le corps de Barry Lyndon est littéralement pénétré. Physiquement et spirituellement épuisé par l'échec d'accéder à la noblesse comme par la mort de son fils, Barry éprouve peu de désir de se venger lorsque Lord Bullingdon le provoque en duel. Quand le pistolet de Bullingdon fait long feu, on accorde à Barry un coup gratuit : il a donc la possibilité de tuer son ennemi et de gagner, enfin, une prise solide sur le domaine Lyndon. Mais au lieu d'éliminer son rival, Barry tire au sol et accorde à Bullingdon un coup gratuit, que ce dernier accepte volontiers. Barry est blessé à la jambe et son chirugien est obligé de la lui amputer.

Exilé d'Angleterre par Bullingdon, séparé de sa femme, sans enfant, sans argent, invalide, Barry devient une sorte de ruine vivante. Il est l'incarnation de la disparition de l'histoire qu'entraîne la tentative même de la récupérer. Ce démembrement est opéré non seulement par le chirurgien, mais par le film lui-même. On sait que pour Derrida, la perte produite par l'écriture dans toutes ses manifestations techniques est une perte positive; qu'elle est la chose qui a disparu dans le désir logocentrique de la présence, et qui ouvre donc au jeu des significations. Mais on ne trouve pas chez Kubrick un tel optimisme; le cinéaste ne perçoit aucune joie dans l'excès de son 
film. Moins nostalgique que mélancolique, Barry Lyndon met au premier plan le caractère exorbitant et la violence du signe cinématographique, afin de dévoiler, dans cette perte, le coût dissimulé de toute production.

\section{Queen's University}

\section{NOTES}

1 Une première version de ce texte a été présentée sous le titre «The Luck of Stanley Kubrick ou la distance», par Lara Fitzgerald, au colloque Écrit/Écran le 1er octobre 1992.

\section{OUVRAGES CITÉS}

Barthes, Roland. «En sortant du cinéma». Communications 23 (1975) pp. 104-107.

Benjamin, Walter. "L'EEuvre d'art à l'ère de sa reproductibilité technique». Essais 2: 1935-1940. Paris : Denoël / Gontier, 1983, pp. 87-126.

Ciment, Michel. Kubrick. Paris : Calmann-Levy, 1980.

Derrida, Jacques. De la grammatologie. Paris : Minuit, 1967.

Derrida, Jacques. La Dissémination. Paris : Seuil, 1972.

Feldman, Hans. «Kubrick and His Discontents». Film Comment 30, 1 (Fall 1976) pp. 12-19.

Gaudreault, André et Jost, François. Le Récit cinématographique. Paris : Nathan, 1990.

Jameson, Frederic. «Progress Versus Utopia; or Can We Imagine the Future?», in Brian Wallis (ed.), Art After Modernism: Rethinking Representation. New York: Godine, 1984, pp. 239-252.

Lukacs, Georges. Le Roman historique. Paris : Payot, 1965.

Oudart, Jean-Pierre. «Barry Lyndon». Cahiers du cinéma 271 (nov. 1976) pp. 62-63.

Stephenson, William. «The Perception of History in Kubrick's Barry Lyndon». Literature / Film Quarterly 9, 1 (1980) pp. 251-259.

Thackeray, William Makepiece. The Luck of Barry Lyndon. New York : New York University Press, 1970. 